University of Nebraska - Lincoln

DigitalCommons@University of Nebraska - Lincoln

Publications, Agencies and Staff of the U.S.

Department of Commerce

U.S. Department of Commerce

$3-2010$

\title{
A Model-Based Approach for Making Ecological Inference from Distance Sampling Data
}

Devin S. Johnson

National Marine Mammal Laboratory

Jeffrey L. Laake

National Marine Mammal Laboratory

Jay M. Ver Hoef

National Marine Mammal Laboratory

Follow this and additional works at: https://digitalcommons.unl.edu/usdeptcommercepub

Part of the Environmental Sciences Commons

Johnson, Devin S.; Laake, Jeffrey L.; and Ver Hoef, Jay M., "A Model-Based Approach for Making Ecological Inference from Distance Sampling Data" (2010). Publications, Agencies and Staff of the U.S. Department of Commerce. 198.

https://digitalcommons.unl.edu/usdeptcommercepub/198

This Article is brought to you for free and open access by the U.S. Department of Commerce at DigitalCommons@University of Nebraska - Lincoln. It has been accepted for inclusion in Publications, Agencies and Staff of the U.S. Department of Commerce by an authorized administrator of DigitalCommons@University of Nebraska - Lincoln. 


\title{
A Model-Based Approach for Making Ecological Inference from Distance Sampling Data
}

\author{
Devin S. Johnson, ${ }^{*}$ Jeffrey L. Laake, and Jay M. Ver Hoef \\ National Marine Mammal Laboratory, Alaska Fisheries Science Center, \\ NOAA National Marine Fisheries Service, Seattle, Washington 98115, U.S.A. \\ *email: devin.johnson@noaa.gov
}

\begin{abstract}
Summary. We consider a fully model-based approach for the analysis of distance sampling data. Distance sampling has been widely used to estimate abundance (or density) of animals or plants in a spatially explicit study area. There is, however, no readily available method of making statistical inference on the relationships between abundance and environmental covariates. Spatial Poisson process likelihoods can be used to simultaneously estimate detection and intensity parameters by modeling distance sampling data as a thinned spatial point process. A model-based spatial approach to distance sampling data has three main benefits: it allows complex and opportunistic transect designs to be employed, it allows estimation of abundance in small subregions, and it provides a framework to assess the effects of habitat or experimental manipulation on density. We demonstrate the model-based methodology with a small simulation study and analysis of the Dubbo weed data set. In addition, a simple ad hoc method for handling overdispersion is also proposed. The simulation study showed that the model-based approach compared favorably to conventional distance sampling methods for abundance estimation. In addition, the overdispersion correction performed adequately when the number of transects was high. Analysis of the Dubbo data set indicated a transect effect on abundance via Akaike's information criterion model selection. Further goodness-of-fit analysis, however, indicated some potential confounding of intensity with the detection function.
\end{abstract}

KEY Words: Abundance; Density; Distance sampling; Line transect; Overdispersion; Spatial point process.

\section{Introduction}

The modern treatment of distance sampling, widely used for estimating plant and animal density, probably began with Eberhardt (1967). The history of this field is dominated by a design-based approach to inference, where the points (locations) are considered fixed and a detection function requires modeling and estimation. Inference is derived from random placement of transects. In contrast, when modeling spatial point patterns all points are assumed to be observed, rather than being fixed, they are assumed to be generated by a random process. The two literatures have remained largely separate from each other. The goals of this article are to (1) combine the ideas of points as coming from a stochastic process with the simultaneous modeling of a detection function in distance sampling and (2) enable inference to abundance estimates and ecological covariates.

Distance sampling is often used to estimate the abundance or density of a population. While traversing a line or at a stationary point, observers record distances from their locations to an object of interest. The main departure from ideal occurs when detection rate of individuals decays as a function of distance from the line. Distance sampling methods have been largely concerned with modeling this decay, termed a detection function (Buckland et al., 2001). In much of the literature, the foundation for inference has remained designbased (e.g., Borchers et al., 1998). Likelihood approaches are used for inference of detection function parameters; e.g., for grouped data (Buckland et al., 2001, p. 108), for imperfect detection on a line (Buckland et al., 2004, p. 108), for doubleobserver counts (Borchers et al., 2006), and for detection covariates (Marques and Buckland, 2003).

Methods for analyzing spatial point patterns have primarily been concerned with learning about the nature of the mechanism that generated the points (e.g., clustered or regular patterns). Recent advances in methodology and computing, however, have focused attention on estimating parameters of spatial point process likelihoods (e.g., Baddeley and Turner, 2000; Møller and Waagepetersen, 2003), which often have difficult form. Textbook treatment may be found in Cressie (1993). In particular, we are interested in the Poisson process (PP; Cox, 1955).

Development of spatially explicit models for distance sampling data has a number of advantages (Hedley and Buckland, 2004; Royle, Dawson, and Bates, 2004) including: (1) coping with opportunistic surveys and surveys with unequal sampling coverage, (2) estimating abundances for specified subregions, and (3) providing a framework for ecological inference for the effects of habitat and other relevant processes on abundance. Schweder (1977) introduced the concept of modeling line transect sampling as thinned point processes, but considered animals to be uniformly distributed (i.e., homogeneous PP). More recently Waagepetersen and Schweder (2006) and Skaug (2006) used likelihood-based methods for line transect sampling, but only estimated parameters of the point process, assuming that the detection function is known. Likewise, Hedley and Buckland (2004) fitted models with a detection 
function that was estimated separately with the distance data (two-stage approach). Simultaneous estimation of detection and abundance allows the inclusion of the uncertainty in detection estimation to be accounted for in the inference of abundance. The reverse is also true; modeled variation in abundance can lead to more efficient detection function inference. Currently, a user of the DISTANCE software (Thomas et al., 2006) can estimate abundance for different regions (e.g., forest/grassland) or ecological treatments (e.g., grazed/not grazed) accounting for variable detection probability but they have rather limited options for evaluating differences or impacts while including the uncertainty and covariances induced from estimation of the detection function.

Simultaneous estimation will also be useful for sampling of small areas or complex habitats (Ramsey and Harrison, 2004) such as surveys of river dolphin (Vidal et al., 1997) or narwhal (Innes et al., 2002) in large bays and narrow fjords. In those cases, rectangular transects with a constant width can extend outside the survey region and the standard uniform distribution assumption for estimation of the detection function is no longer valid. Those situations can be partially accommodated by allowing variation in transect width and using spatial stratification (Dawson et al., 2004) or by adjusting the likelihood for fitting the detection function (Laake et al., 2008), but simultaneous estimation is a more natural formulation. Royle et al. (2004) proposed an integrated likelihood for simultaneous estimation of detection and abundance from point count data but it only allowed site-based effects on average detection probability. In this article, we take a full likelihood-based approach for simultaneous estimation of parameters of the detection function and the (in)homogeneous point process, which provides a framework for ecological inference from distance sampling data and accommodates sampling of small or complex habitats.

The remainder of the article is organized as follows. In Section 2, we lay out the basic models and notation. In Section 3, we consider inhomogeneous point patterns and concentrate on inference for covariates and abundance, including the expected abundance and realized abundance. Section 4 gives some simulated and real examples. We conclude with a discussion in Section 5.

\section{Likelihood Formulation for Distance Sampling}

For the sake of exposition, let us assume we are talking about ecological inference on abundance in a single contiguous study area, say $A$, with distinct spatial boundaries. The theory for sampling $A$ with transects versus points as observation platforms are equivalent; without loss of generality we will consider sampling individuals from transects.

\subsection{Modeling Ecological Influences on Abundance}

To begin a model-based approach, we assume that locations $\mathbf{s}=\left(s_{x}, s_{y}\right)$ of all individuals in $A$, say $\mathcal{S}^{+}=\left(\mathbf{s}_{1}, \ldots, \mathbf{s}_{N}\right)$, is a realization of a PP with intensity function $\lambda(\mathbf{s}, \boldsymbol{\beta})=$ $\exp \left\{\mathbf{x}(\mathbf{s})^{\prime} \boldsymbol{\beta}\right\}$, where $\mathbf{x}(\mathbf{s})$ is a $p$-vector of concomitant environmental variables $\left(x_{1}(\mathbf{s}) \equiv 1\right)$ measured at $\mathbf{s}$ and $\boldsymbol{\beta}$ a $p$-vector of parameters. Other forms of $\lambda$ can certainly be used depending on the situation.
If all of the individuals could be located within $A$, then inference about $\boldsymbol{\beta}$ could be made by maximizing the PP log likelihood

$$
\ell_{P P}\left(\boldsymbol{\beta} ; \mathcal{S}^{+}\right)=\sum_{i=1}^{N} \mathbf{x}\left(\mathbf{s}_{i}\right)^{\prime} \boldsymbol{\beta}-\int_{A} \exp \left\{\mathbf{x}(\mathbf{u})^{\prime} \boldsymbol{\beta}\right\} d \mathbf{u} .
$$

This is not the case, however, either $A$ cannot be surveyed in entirety or individuals are missed during survey. Usually both of these departures from an ideal census are assumed. Next, we incorporate these into the analysis.

\subsection{Incorporating Uncertain Detection}

To make use of the PP model for inference with distance sampling data we modify the standard PP likelihood (1) to allow for the fact that $A$ is not surveyed in its entirety and individuals are not detected with certainty. This can be accomplished by viewing the line transect sampling procedure as thinning the original location process $\mathcal{S}^{+}$to obtain the locations of observed individuals $\mathcal{S}=\left(\mathbf{s}_{1}, \ldots, \mathbf{s}_{n}\right)$. Thinning the location process involves a supplementary function $q(\mathbf{s}): A \rightarrow[0,1]$. For a given realization of locations $\mathcal{S}^{+}$one thins the process by retaining each point with probability $q\left(\mathbf{s}_{i}\right)$ and discards the rest to obtain the subset $\mathcal{S}$. The resulting intensity function of the thinned PP $\mathcal{S}$ is $q(\mathbf{s}) \lambda(\mathbf{s} ; \boldsymbol{\beta})$ (Cressie, 1993, p. 691). To formulate the thinning notion for distance sampling, we assume, without loss of generality, that $A$ is surveyed in disjoint regions $C_{k} \subset A ; k=1, \ldots, K$. Herein, we will deal with straight line transect corridors with width $2 w_{k}$.

In distance sampling methodology the individuals present in the corridors are detected at a rate $g(\mathbf{s} ; \cdot)$. We assume the following general form for the detection function,

$$
g\left(\mathbf{s} ; \alpha_{k}, \gamma\right)=\exp \left[-\left\{z_{k}(\mathbf{s}) / \alpha_{k}\right\}^{1 / \gamma}\right]
$$

where, for $\mathbf{s} \in C_{k}, z_{k}(\mathbf{s})$ is the perpendicular distance from a location at $\mathbf{s}$ to the transect center line, otherwise, $g\left(\mathbf{s} ; \alpha_{k}\right.$, $\gamma) \equiv 0$. This form encompasses many traditional functions in Buckland, Anderson, et al. (1993) (Gaussian, $\gamma=0.5$; exponential $\gamma=1$; uniform $\gamma \rightarrow 0$ ). By defining (2) for every location in $A$, we obtain the necessary thinning function,

$$
q(\mathbf{s} ; \boldsymbol{\eta})=\sum_{k=1}^{K} g\left(\mathbf{s} ; \alpha_{k}, \gamma\right)
$$

where $\boldsymbol{\eta}=\left(\alpha_{1}, \ldots, \alpha_{K}, \gamma\right)^{\prime}$.

Using the thinning function we can now define a full likelihood for distance sampling by multiplying $\lambda(\mathbf{s}, \boldsymbol{\beta})$ by $q(\mathbf{s}, \boldsymbol{\eta})$ to obtain the likelihood for the observed data $\mathcal{S}$,

$$
\begin{aligned}
\ell_{D S}(\boldsymbol{\theta} ; \mathcal{S})= & \sum_{k=1}^{K} \sum_{j=1}^{n_{k}}\left[\mathbf{x}\left(\mathbf{s}_{j}\right)^{\prime} \boldsymbol{\beta}-\left\{z_{k}\left(\mathbf{s}_{j}\right) / \alpha_{k}\right\}^{1 / \gamma}\right] \\
& -\sum_{k=1}^{K} \int_{C_{k}} \exp \left[\mathbf{x}(\mathbf{u})^{\prime} \boldsymbol{\beta}-\left\{z_{k}(\mathbf{u}) / \alpha_{k}\right\}^{1 / \gamma}\right] d \mathbf{u},
\end{aligned}
$$

where $n_{k}$ is the number of animals detected in $C_{k}$ and $\boldsymbol{\theta}=$ $\left(\boldsymbol{\beta}^{\prime}, \boldsymbol{\eta}^{\prime}\right)^{\prime}$.

The likelihood in equation (3) is a generalization of the likelihood given by equation (2.9) in Hedley and Buckland (2004). Hedley and Buckland use a thinned PP likelihood with 
the additional assumptions that (1) $\lambda(\mathbf{s}, \boldsymbol{\beta})$ is a function of $\mathbf{s}$ only in terms of the parallel length along the transect centerline ("waiting time till detection"), (2) transect width is constant within and between all transects. In addition, Hedley and Buckland do not simultaneously estimate intensity and detection parameters.

\section{Ecological Inference}

There are two types of inferences that one would like to make with the distance sampling model given in Section 2.2, determination of the relationship between ecological covariates and distribution of individuals in $A$ and estimating the abundance of individuals in a region $B \subseteq A$. First, we will examine the former via parameter inference, then, the latter via a prediction-type inference.

\subsection{Parameter Estimation}

Using the likelihood (3) derived in Section 2.2, we consider maximum likelihood estimation (MLE), which generally provides asymptotically normal and efficient estimators. This is also the case in equation (3). Suppose the number of transects $K$ is allowed to become large such that $\cup_{k=1}^{K} C_{k} \rightarrow \mathbb{R}^{2}$, and elements of $\mathbf{x}(\mathbf{s})$ are not highly collinear with $z_{k}(\mathbf{s})$ on all transects. Then, the sampling distribution of the MLE $\hat{\boldsymbol{\theta}}$ approaches normality with the process generating parameters, say $\boldsymbol{\theta}^{*}$, as the mean and variance equal to

$\boldsymbol{\Sigma}=\left[\sum_{k=1}^{K} \int_{C_{k}} \mathbf{h}_{k}(\mathbf{u}) \mathbf{h}_{k}(\mathbf{u})^{\prime} \exp \left[\mathbf{x}(\mathbf{u})^{\prime} \boldsymbol{\beta}^{*}-\left\{z_{k}(\mathbf{u}) / \alpha_{k}^{*}\right\}^{1 / \gamma}\right] d \mathbf{u}\right]^{-1}$

where $\mathbf{h}_{k}(\mathbf{s})=\left[x_{1}(\mathbf{s}), \ldots, x_{p}(\mathbf{s}), \mathbf{d}_{k}^{\prime}(\mathbf{s})\right]^{\prime}$ and $\mathbf{d}_{k}(\mathbf{s})$ is a $K$ vector with zeros at all entries but the $k$ th which is equal to $\left\{z_{k}(\mathbf{s}) / \alpha_{k}^{*}\right\}^{1 / \gamma} / \gamma \alpha_{k}^{*}$. This is a result of Theorem 1 in Guan and Loh (2007). Here, for ease of exposition, we assumed $\gamma$ to be fixed and let it be used as a model definition. Typically equation (3) has to be maximized numerically, but if $\lambda(\mathbf{s}, \boldsymbol{\beta})=\lambda$ (i.e., a homogeneous PP), then estimates can be found analytically and correspond to some traditional designbased distance sampling estimators (see Web Appendix). In addition to efficient estimation, ecological inference on covariate selection can be made using model selection methods, such as Akaike's information criterion (AIC; Burnham and Anderson, 2002).

\subsection{Estimating Abundance}

In addition to the parameters themselves, another quantity of interest is the abundance in a particular subregion $B \subseteq$ $A$. Because the distribution of individuals is random under the model-based paradigm, there are two types of abundance that we will consider. First, is the expected abundance, $\mu(B ; \boldsymbol{\beta})=\int_{B} \lambda(\mathbf{u}, \boldsymbol{\beta}) d \mathbf{u}$. The second type is the realized abundance $N(B)=\mathcal{S}^{+} \cap B$ for a given realization of $\mathcal{S}^{+}$from $\lambda(\mathbf{s}, \boldsymbol{\beta})$. The value $N(B)$ is a Poisson random variable with mean $\mu(B ; \boldsymbol{\beta})$. Over several surveys one would expect to see, on average, $\mu(B ; \boldsymbol{\beta})$ individuals in $B$. For a single survey, however, there were $N(B)$ individuals present at that particular time. The quantity $N(B)$ is analogous to prediction of an observation and $\mu(B ; \boldsymbol{\beta})$ is a trend.
We begin with expected abundance estimation. Through standard theory, the MLE of expected abundance is

$$
\mu(B ; \hat{\boldsymbol{\beta}})=\int_{B} \exp \left\{\mathbf{x}(\mathbf{u})^{\prime} \hat{\boldsymbol{\beta}}\right\} d \mathbf{u}
$$

Using the delta method (Dorfman, 1938), the large sample variance of $\mu(B ; \hat{\boldsymbol{\beta}})$ is approximately

$$
\operatorname{Var}\{\mu(B ; \hat{\boldsymbol{\beta}})\} \approx \mathbf{b}^{\prime} \mathbf{\Sigma} \mathbf{b},
$$

where the ith entry of $\mathbf{b}$ is

$$
b_{i}=\int_{B} x_{i}(\mathbf{u}) \exp \left\{\mathbf{x}(\mathbf{u})^{\prime} \boldsymbol{\beta}^{*}\right\} d \mathbf{u}, \quad i=1, \ldots, p,
$$

for $p$ covariate parameters and $b_{i}=0$ for the remaining $K$ $\alpha$-parameters. The most straightforward variance estimator results from substituting $\hat{\boldsymbol{\theta}}$ for $\boldsymbol{\theta}^{*}$ in equation (4).

We now turn our attention to estimation of the realized abundance, $N(B)$. Before obtaining the proposed estimator, we first note that $N(B)=n(B)+N_{u}(B)$, where $N_{u}(B)$ is the number of undetected individuals in $B$ and $n(B)$ is the number of observed individuals. The number of unobserved animals is a Poisson random variable with expectation

$$
\xi\left(B ; \boldsymbol{\theta}^{*}\right)=\int_{B}\left\{1-q\left(\mathbf{u} ; \boldsymbol{\eta}^{*}\right)\right\} \exp \left\{\mathbf{x}(\mathbf{u})^{\prime} \boldsymbol{\beta}^{*}\right\} d \mathbf{u} .
$$

Moreover, given any value $\boldsymbol{\theta}^{*}, N_{u}(B)$ is independent of $n(B)$.

The first predictor of $N(B)$ that comes to mind turns out to be asymptotically efficient. By substituting $\hat{\boldsymbol{\theta}}$ for $\boldsymbol{\theta}^{*}$ in equation (5) one obtains the predictor $\hat{N}(B)=n(B)+\xi(B ; \hat{\boldsymbol{\theta}})$. The mean square prediction error (MSPE) for $\hat{N}(B)$ is given by

$$
\begin{aligned}
\operatorname{MSPE}\left\{\hat{N}(B) ; \boldsymbol{\theta}^{*}\right\}= & \left.E\left[\{\hat{N}(B)-N(B)\}^{2} ; \boldsymbol{\theta}^{*}\right\}\right] \\
= & \xi\left(B ; \boldsymbol{\theta}^{*}\right)+\operatorname{Var}\left\{\xi(B ; \hat{\boldsymbol{\theta}}) ; \boldsymbol{\theta}^{*}\right\} \\
& +\operatorname{Bias}\left\{\xi(B ; \hat{\boldsymbol{\theta}}) ; \boldsymbol{\theta}^{*}\right\}^{2} \\
\approx & \xi\left(B ; \boldsymbol{\theta}^{*}\right)+\mathbf{c}^{\prime} \mathbf{\Sigma} \mathbf{c},
\end{aligned}
$$

where the $i$ th element of $\mathbf{c}$ is

$$
c_{i}= \begin{cases}\int_{B} x_{i}(\mathbf{u})\left\{1-q\left(\mathbf{u} ; \boldsymbol{\eta}^{*}\right)\right\} & i=1, \ldots, p, \\ \times \exp \left\{\mathbf{x}(\mathbf{u})^{\prime} \boldsymbol{\beta}^{*}\right\} d \mathbf{u} ; & i=p+1, \ldots, p+K . \\ \sum_{k=1}^{K} \int_{B \cap C_{k}} \frac{1}{\gamma \alpha_{k}^{*}}\left\{\frac{z_{k}(\mathbf{u})}{\alpha_{k}^{*}}\right\}^{1 / \gamma} & i=1, k\end{cases}
$$

The last step in equation (6) results from the fact that asymptotically $\operatorname{Var}\left\{\xi(B ; \hat{\boldsymbol{\theta}}) ; \boldsymbol{\theta}^{*}\right\} \rightarrow \mathbf{c}^{\prime} \boldsymbol{\Sigma} \mathbf{c}$ and $\hat{\boldsymbol{\theta}}$ is a consistent estimator. Again, replacing $\boldsymbol{\theta}^{*}$ by $\hat{\boldsymbol{\theta}}$ in equation (6) provides an estimator of the MSPE.

There are two notes concerning equation (6). First, application of Theorem 1 in Nayak (2002) shows that the last line is the lower bound for MSPE. Therefore, $\hat{N}(B)$ is asymptotically most efficient. Second, as $B \cap C_{k} \rightarrow B$ and $\alpha_{k}^{*} \rightarrow$ $\infty$ for all transects, then we count all animals in $B$, and $\operatorname{MSPE}\left\{\hat{N}(B) ; \boldsymbol{\theta}^{*}\right\} \rightarrow 0$, as it should. Thus, a finite population correction factor is automatically embedded in the variance estimator. This bypasses the issue of an ad hoc decision on a 
finite population correction factor as discussed in Buckland, Anderson, et al. (1993, p. 96).

\subsection{Overdispersion}

Small-scale variation in the intensity function that is unexplained by the spatial covariates may affect local abundance estimates as well as variance estimates. Overdispersion of count-like data is a common occurrence in ecological data sets. Waagepetersen and Schweder (2006) and Skaug (2006) propose a point process model specifically designed for clustered data. Both make use of a Cox process (Diggle, 2003) to model clustering behavior, which leads to overdispersed abundance. Waagepetersen and Schweder (2006) use computationally intensive sampling algorithms to obtain parameter estimates for a homogeneous Cox process (single average intensity for the entire study area).

We investigate another, admittedly ad hoc, procedure for accounting for overdispersion that is much less computationally intensive. Our proposal involves calculation of the overdispersion factor

$$
\begin{aligned}
\hat{c} & =\min \left\{1,\left[\sum_{k=1}^{K} \frac{\left\{n\left(C_{k}\right)-\hat{E}_{k}\right\}^{2}}{\hat{E}_{k}} /(K-m)\right]^{-1 / 2}\right\} \\
& =\min \left\{1,\left(\chi^{2} / d f\right)^{-1 / 2}\right\},
\end{aligned}
$$

where $m$ is the number of parameters and $\hat{E}_{k}=$ $\int_{C_{k}} g_{k}(\mathbf{u} ; \hat{\alpha}) \lambda(\mathbf{u} ; \hat{\boldsymbol{\beta}}) d \mathbf{u}$ is the estimated expected number of observed animals in transect $k=1, \ldots, K$. This is motivated by the overdispersion correction for a Poisson generalized linear model (McCullagh and Nelder, 1989, p. 127). All standard errors can then be divided by $\hat{c}$ to inflate them and correspondingly widen the associated confidence intervals. There are two main benefits to this method: (1) computation is very simple after the model has been fitted via MLE and (2) the correction is nonparametric in that no model for a Cox process is necessary. The choice of transects as the basis for measuring overdispersion is somewhat arbitrary. But, it is a well-defined unit in every distance sampling analysis and is often the unit used for increasing "sample size." See Sections 4.2 and 5, however, for alternatives and discussion.

\section{Examples}

In this section, we present a simulation experiment, as well as analysis of the Dubbo weed data originally analyzed by Melville and Welsh (2001). All of the simulated data generation and analysis in this section were performed using a package called DSpat that we have developed to implement point process modeling of distance sampling data in the $\mathrm{R}$ language (R Development Core Team, 2008). DSpat will be available on CRAN (http://cran.r-project.org/) and it contains the weed data and the analysis we present here. The DSpat package depends heavily on the package spatstat (Baddeley and Turner, 2005, see http://www.spatstat.org) and to a lesser degree the packages gpclib, RandomFields (Schlather, 2001), and mgcv (Wood, 2006). All are available at http://www.rproject.org. DSpat uses the quadrature scheme of Berman and Turner (1992) for calculation of the likelihood (3).

\subsection{A Simulation Experiment}

We conducted a simulation study to validate the analysis technique and the $\mathrm{R}$ package we developed using simulated data from a systematic sampling design with known model structures for process intensity and detection. We also evaluated confidence interval coverage under scenarios with and without overdispersion and compared the results to a conventional distance sampling (CDS) estimator (Buckland et al., 2001) with assumption of random line placement (CDS-R2) and a modified, more appropriate, version (CDS-O1) for systematic line placement (Fewster et al., 2009).

We simulated point data over a square $100 \times 100$ study area with a homogeneous PP and an inhomogeneous PP (IPP) with and without overdispersion. The structure for the IPP included three habitat types with varying intensity and a vertical linear feature (e.g., river) in the center of the study area with decreasing intensity to the east and west of center. The habitat features were constructed by generating a smooth Gaussian random field across the study area and then defining the habitats based on quantiles of $33.3 \%$ and $66.7 \%$ to provide an approximately equal area for each habitat (Figure 1). The $\log$ of intensity across the surface was defined as $x(\mathbf{s})^{\prime} \boldsymbol{\beta}$, where $\boldsymbol{\beta}=\left(\beta_{1}, 1,2,-1\right)$ and $x_{1}(\mathbf{s})=1, x_{2}(\mathbf{s})$ and $x_{3}(\mathbf{s})$ are $0 / 1$ dummy variables for habitats 2 and 3 , and $x_{4}(\mathbf{s})=\mid s_{x}-$ $50 \mid / 100$, a scaled horizontal distance from $\mathbf{s}$ to the center. The vertical transect lines were systematically spaced and the grid was given a random starting position relative to the study area. The width of each transect was computed as $w_{k}=$ $100 P / K$, where $P$ is the proportion of the study area sampled and $K$ is the number of transects. Any portion of the transect that extended outside of the study area was excluded, so transect width could vary for at least one transect depending on the coverage and random placement of the grid. A halfnormal detection function (equation $(2) ; \gamma=0.5$ ) was used for observation of points. The scale parameter $\alpha$ was computed such that the average detection probability, say $\bar{g}$ was either 0.25 or 0.6 in a simulation scenario. Overdispersion was incorporated by modeling the intensity with a log-Gaussian Cox process (LGCP), $\log \lambda(\mathbf{s} ; \boldsymbol{\beta})=\mathbf{x}(\mathbf{s})^{\prime} \boldsymbol{\beta}+\epsilon(\mathbf{s})$, where $\epsilon(\mathbf{s})$ was a Gaussian random field with correlation function $\operatorname{cov}[\epsilon(\mathbf{s})$, $\epsilon(\mathbf{s}+\mathbf{h})]=\tau^{2} \exp \left(-\|\mathbf{h}\|^{2} / \phi\right), \quad \tau=0.5$, and $\phi=-5^{2} /$ $\log (0.05)$. These values give a range of spatial correlation of approximately five units (correlation $\leq 0.05$ beyond five units) and variance of 0.25 for the $\epsilon(\mathbf{s})$ process. For all simulated data sets the intercept $\beta_{1}$ was adjusted so that the expected number of observed individuals $E(n)=E(N) P \bar{g}=$ 75 or 400 depending on the scenario.

For each of the 48 scenarios (homogeneous Poisson, inhomogeneous Poisson with and without overdispersion; $P=$ $0.04,0.50 ; K=10,20 ; \bar{g}=0.25,0.60 ; E(n)=75,400), 1825$ replicate simulations were conducted in which intensity (habitat), point locations, lines, and detection were randomized for each replicate. The number of replicate simulations was chosen so the empirical confidence interval coverage should be within $\pm 1 \%$ of the actual $95 \%$ coverage.

Here, we present results for the estimation of the expected abundance $\mu(A)$ only. The results were nearly identical for estimation of the realized abundance $N(A)$. In each of the 48 scenarios, the estimated bias never exceeded $1.5 \%$ and the 
True Intensity

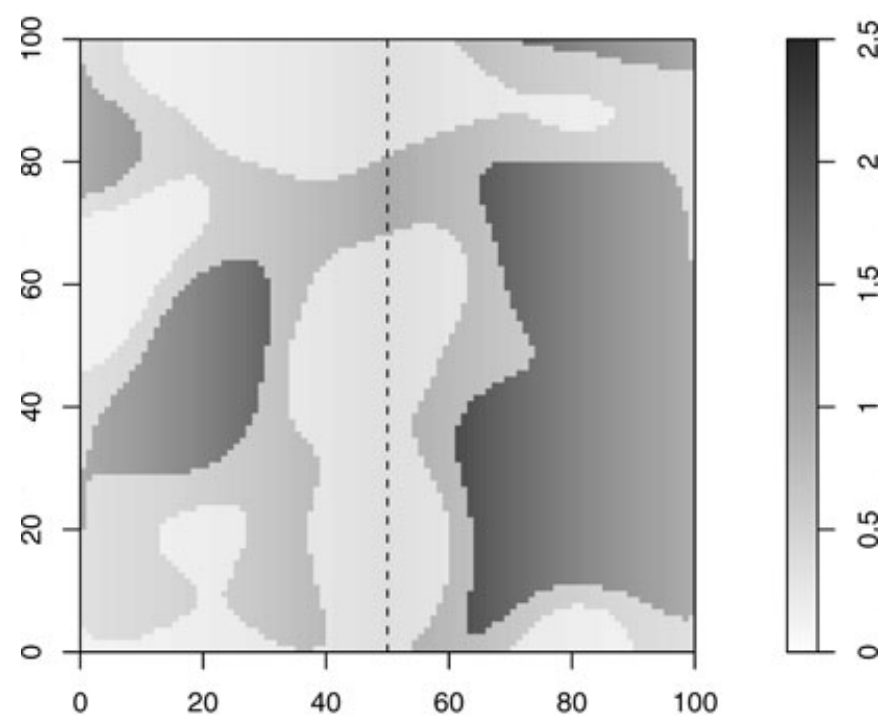

Estimated Intensity

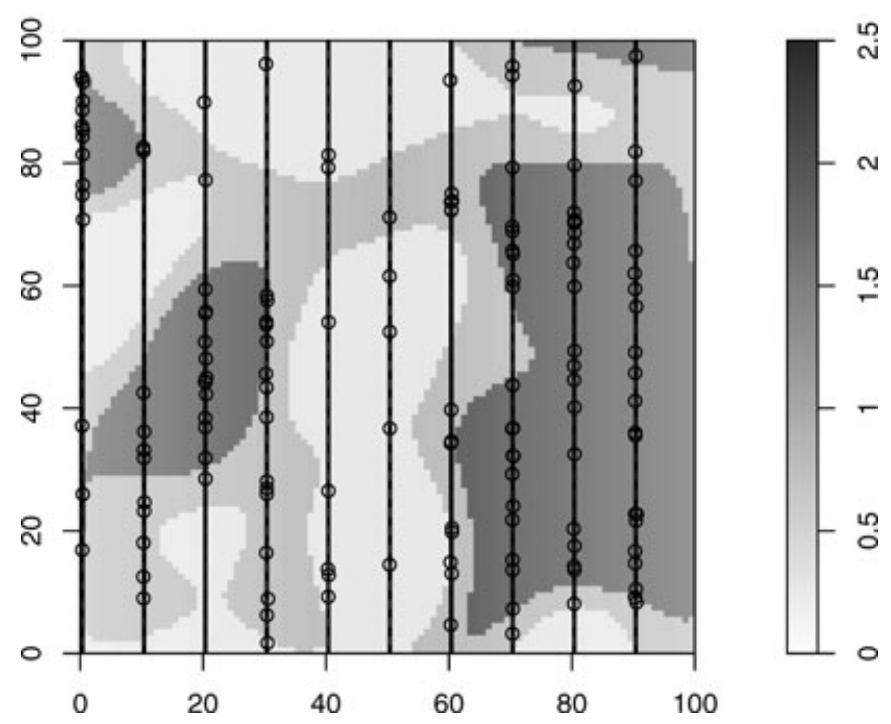

Figure 1. Example simulated intensity surface (top) and estimated (bottom) surface with habitat and river feature and $K=10, \bar{g}=0.25, P=4 \%$, and $E(n)=75$.

average across all scenarios was $0.9 \%$ and $0.08 \%$ for $E(n)=$ 75 and 400, respectively. We expect that any small amount of bias that does exist would occur from the resolution of the quadrature points for integration. Figure 1 illustrates that even with sparse transect sampling, informative gridded environmental covariates can lead to an accurate prediction of intensity over the entire region with a known model.

The confidence interval coverage for the homogeneous Poisson scenarios was within the expected range for both the CDS and model-based (IPP) estimators (Figure 2). For the IPP scenarios without overdispersion, the confidence interval coverage for the model-based estimator was within the expected range but the observed coverage for CDS was too high except for the case with small sample size $(E(n)=75)$ and larger number of lines $(K=20)$. When overdispersion was added, the observed coverage was even higher for CDS. For the model-based estimator the ad hoc adjustment for overdispersion was not sufficient and coverage was too low except with the larger number of lines $(K=20)$ and $4 \%$ sampling coverage. For most real applications, sampling coverage is less than $4 \%$, so the ad hoc adjustment may be adequate as long as $K$ is sufficiently large.

The average estimated coefficient of variation for the CDSO1 variance estimator was $24 \%$ larger than the coefficient of variation for the model-based estimator for the IPP process without overdispersion. This reflected the bias in the estimated standard error for CDS-O1, which was $23 \%$ larger than the standard deviation of the replicate values of $\mu(A)$. The CDS-O1 variance estimator provided a reduction of $25 \%$ in comparison to the CDS-R2 estimator but was still larger than the true variance. Thus, as long as overdispersion can be accommodated, the model-based estimator can provide substantial gains in precision in comparison to CDS even with the more recently developed systematic variance estimators.

\subsection{Application to the Dubbo Weed Data}

Melville and Welsh (2001) collected and analyzed line transect sampling data with $n=479$ observed devil's claw weeds in a farming paddock from eight $150 \mathrm{~m}$ wide parallel transects. These data are analyzed here as an example because the entire population was enumerated and the data highlight problems that can occur in some unusual circumstances. Melville and Welsh (2001) stated that signed perpendicular distance $\left(z_{k}(\mathbf{s})\right)$ and the distance along each transect were measured; however, only the signed perpendicular distances were provided and they make no mention of the transect length in their paper. To analyze these data in a spatial context we have assumed that the paddock was square (1200 m by $1200 \mathrm{~m}$ ) and we generated a restricted random uniform $s_{y}$ coordinate for each object such that no weeds had the exact same coordinate. There were 742 weeds in the paddock with 99, 136, $90,102,54,66,101$, and 91 in transects $1-8$, respectively (Melville and Welsh, 2001). They stated that sheep were only present on transects 5-8 and they ate the leafy part of the weed so they expected that the weeds would be harder to detect on those transects. Using the known positions of all weeds and the observed weeds, we can describe the actual distribution of all perpendicular distances and the proportion detected within intervals of distance for transects with sheep absent and present (Table 1). Detection probability was slightly lower where sheep were present, but also the frequency of perpendicular distances decreased with distance where sheep were absent and increased with distance where sheep were present. This resulted in declining numbers observed with distance where sheep were absent and a roughly constant number observed for each interval where sheep were present.

We fitted models in which weed intensity differed (1) for sheep absence/presence, (2) for each of the eight strips, and (3) as a thin-plate regression spline (Wood, 2006) of the eastwest coordinate $s_{x}$. We modeled detection probability within the strip as a half-normal function (equation $(2) ; \gamma=0.5$ ) 

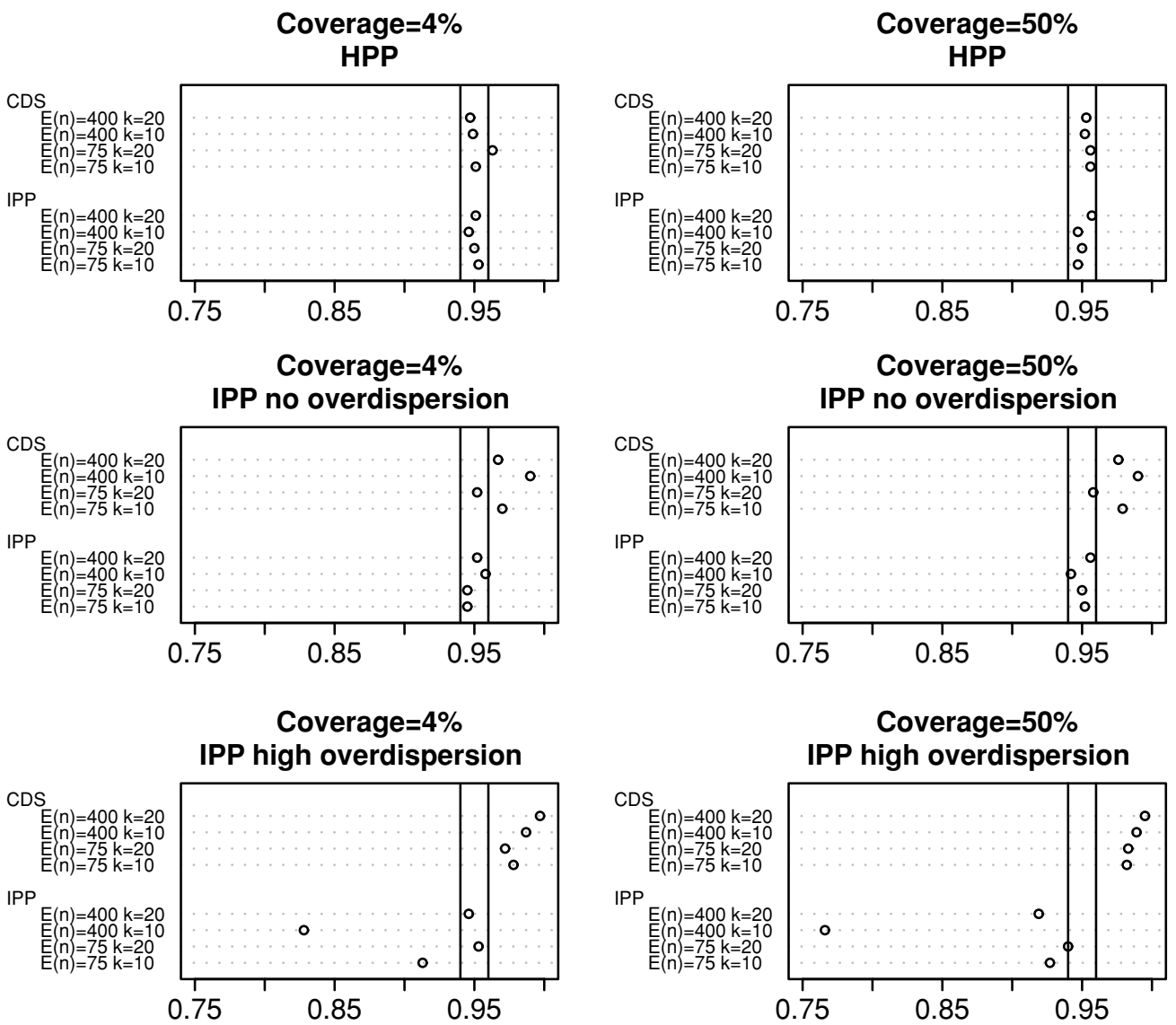

Figure 2. Confidence interval coverage (nominal 95\%) for 1825 replicates with the IPP and CDS-O1 estimators for each scenario with $\bar{g}=0.25$. Results for $\bar{g}=0.60$ were very similar. Vertical bars show expected range of simulation error.

Table 1

Known number of weeds $(N)$ and the proportion $(p)$ and number $(n)$ observed in 10 equal distance bins for transects 1-4 (sheep absent) and transects 5-8 (sheep present)

\begin{tabular}{|c|c|c|c|c|c|c|c|c|c|c|c|}
\hline Sheep & & {$[0,7.5]$} & $(7.5,15]$ & $(15,22.5]$ & $(22.5,30]$ & $(30,37.5]$ & $(37.5,45]$ & $(45,52.5]$ & $(52.5,60]$ & $(60,67.5]$ & $(67.5,75]$ \\
\hline \multirow{3}{*}{ Absent } & $N$ & 57 & 90 & 32 & 43 & 37 & 49 & 54 & 27 & 11 & 30 \\
\hline & $p$ & 1.00 & 0.97 & 1.00 & 0.81 & 0.86 & 0.86 & 0.63 & 0.37 & 0.18 & 0.33 \\
\hline & $n$ & 57 & 87 & 32 & 35 & 32 & 42 & 34 & 10 & 2 & 10 \\
\hline \multirow[t]{3}{*}{ Present } & $N$ & 11 & 21 & 16 & 17 & 36 & 48 & 46 & 49 & 31 & 37 \\
\hline & $p$ & 1.00 & 0.81 & 0.75 & 0.76 & 0.50 & 0.42 & 0.35 & 0.31 & 0.26 & 0.22 \\
\hline & $n$ & 11 & 17 & 12 & 13 & 18 & 20 & 16 & 15 & 8 & 8 \\
\hline
\end{tabular}

and considered a model with constant $\alpha$ and another in which $\alpha$ differed based on sheep presence and absence. The model with minimum AIC included a separate $\alpha$ for sheep presence/absence and intensity varying across strips (Table 2). The known population size was within one standard error of the estimated value of 774 . However, the model was unable to reflect the true spatial distribution of weeds across the paddock (Figure 3). The estimated number of weeds in transects 1-4 (sheep absent) were too high and the estimates were too low for transects 5-8 (sheep present). This occurred because the true spatial intensity was not constant within the strips and it varied based on presence of sheep. The estimated value $\hat{\alpha}$ was 23.6 where sheep were absent and was substantially larger at 56.5 where sheep were present. The latter value should have been smaller because detection probability declined more rapidly where sheep were present. This is apparent by examining the observed and expected distributions for perpendicular distance (Figure 4). Using the distance bins in Figure 4 instead of transects, a $\chi^{2}$ statistic can be calculated as in equation (7). There is a substantial lack of fit $\left(\chi^{2}=\right.$ $51.5, d f=10, p<0.001)$ and the residuals reflect the increasing frequency of weeds with distance for transects with sheep present. The distance bin calculated $\hat{c}$ approximately doubles the standard errors for the abundance estimates in Table 2.

Even though the modeled intensity surface showed substantial lack of fit in an absolute (but not qualitative) sense, we chose the Dubbo data to illustrate not only the ability to compare environmental treatments with the IPP method, but 
Table 2

Models fitted to Dubbo weed data and resulting estimates and precision of weed abundance in the entire paddock

\begin{tabular}{llcrcc}
\hline \hline Intensity $^{\text {a }}$ & Detection & \# parameters & $\Delta$ AIC & $\hat{N}$ & Std. error \\
\hline$\sim$ Sheep & $\sim 1$ & 3 & 38.1 & 755 & 46 \\
$\sim$ Sheep & $\sim$ Sheep & 4 & 15.8 & 773 & 47 \\
$\sim$ Strip & $\sim 1$ & 9 & 22.4 & 755 & 46 \\
$\sim$ Strip & $\sim$ Sheep & 10 & 0.0 & 774 & 47 \\
$\sim$ S $(x)$ & $\sim 1$ & 11 & 18.9 & 760 & 47 \\
$\sim$ S $(x)$ & $\sim$ Sheep & 12 & 5.2 & 769 & 47 \\
\hline
\end{tabular}

${ }^{\mathrm{a}} \mathrm{S}(x)$ denotes thin-plate regression spline model.

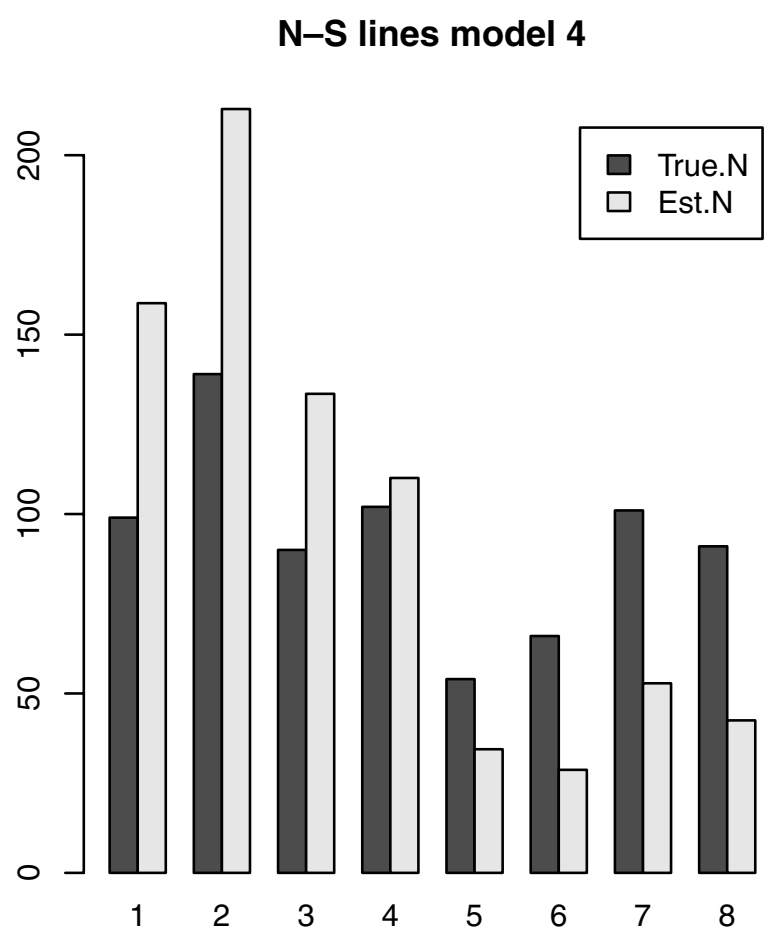

Figure 3. True (dark) and estimated (light) number of weeds in each strip using the minimum AIC model for the Dubbo weed data.

also the ability to explore where or why a hypothesized relationship may not fit as expected. We refrain from speculation here, but a researcher using this data can now explore the lack of fit and possibly look for other covariates which might be associated with this discrepancy. Certainly sheep presence is not enough to fully explain abundance patterns in this data.

\section{Discussion}

Casting distance sampling into a full likelihood formulation for the spatial point process and the observation process (detection) provides a natural model selection framework to evaluate impacts of ecological processes and experimental manipulations (e.g., grazing) on animal abundance and distribution. Simultaneous fitting of the detection function copes with the potential influence of those same ecological variables and others (e.g., observer) on the point process parameter estimates

\section{Sheep absent}

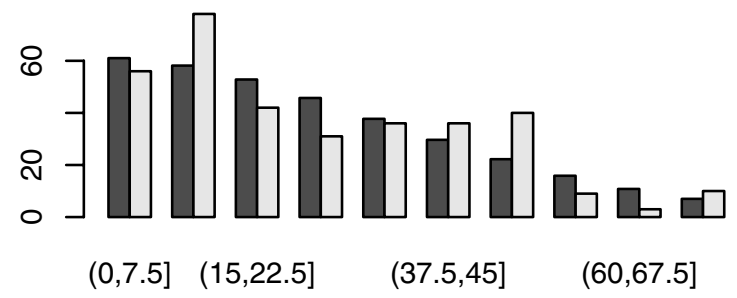

Sheep present

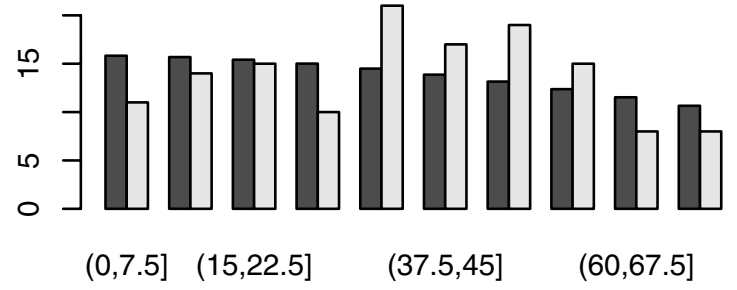

Figure 4. Observed (light) and expected (dark) numbers of weeds in perpendicular distance intervals for transects with sheep absent and present.

and uncertainty resulting from the estimation of the detection parameters.

Use of this model-based approach does not require random transect placement so it will work as well with systematic designs, platforms of opportunity, and more optimal designs that are not restricted to designs with transects parallel to the density gradient. However, some degree of caution is warranted and design considerations cannot be completely ignored. It is possible to pose models in which the detection parameters are completely confounded with the intensity parameters. For example, a model in which the underlying point process is a symmetric function of the perpendicular distance from the line is completely confounded with the detection process. Confounding of detection probability and intensity with this model-based approach can be avoided in a variety of ways. Melville and Welsh (2001) proposed estimation of detection from a calibration strip in which all objects were delineated and detection or nondetection was determined for each object. Detection probability would then be assumed to be constant across all of the strips. This is a rather strong assumption and the method would be impractical and inefficient in most situations. An alternative and more efficient approach would be a pattern of perpendicular strips as described by Buckland et al. (2007), which would enable detection of objects in both the $s_{x}$ and $s_{y}$ directions with certainty. Another alternative is to survey with two independent observers allowing detection probability to be independently assessed with the capturerecapture data (Laake and Borchers, 2004; Borchers et al., 2006). This latter approach was used in migration counts of gray whales (Buckland, Breiwick, et al., 1993) in which the true offshore distribution (intensity) is confounded with the detection process in the context of distance sampling. Alternatively, aerial surveys perpendicular to shore could be used to model the intensity process as a function of distance from 
shore which would eliminate any confounding as suggested by Buckland et al. (2007).

Models for the spatial point process can be limited to use spatial coordinates like the one-dimensional spline used in the Dubbo weed example. Similar models can be created to provide a smooth two-dimensional surface for the point process intensity. However, many practitioners will want to include habitat covariates or experimental treatments and such models can be easily created. There is one drawback in using spatial covariates when the goal is estimation of total abundance. Unlike design based methods, the spatial covariates need to be known for the entire region and not just in the sampled region. Sometimes specifying the spatial covariates is a trivial exercise like the sheep treatment in the Dubbo weed data example. But in most cases, the spatial covariate values will need to be defined from a raster grid applied to layers of a geographical information system. The tools needed to manipulate geographical information system layers are available as packages for the $\mathrm{R}$ statistical software ( $\mathrm{R}$ Development Core Team, 2008) that can be integrated with the DSpat package.

There are two nontrivial extensions to the present work that would enhance the model-based method. First, our ad hoc approach for overdispersion is admittedly not a perfect solution because it will depend on the choice of scale and is limited by the degrees of freedom which can be nonpositive if too many parameters are fitted. The computationally intensive approach of Waagepetersen and Schweder (2006) is also limited by scale and can only measure overdispersion at the level of half of the transect width as well as requiring a Cox process model be specified. A potential modification would be to use a one-dimensional $K$-function along the line to increase the potential scale for overdispersion measurement. Second, extensions of this work to include marked point processes would be useful for handling animals in groups (e.g., pods, flocks, or herds) and to examine species interactions. Using Markov point process models might be useful for this case. Markov models can be fitted using a pseudo-likelihood function with little change to equation (3). If ecological inference is the primary goal, then this may suffice; however, it is nontrivial to incorporate interaction into abundance estimation. The same is true of the Cox process. Clearly, this is an area that needs further research.

\section{Supplementary Material}

The Web Appendix referenced in Section 3.1 is available under the Paper Information link at the Biometrics website http://www.biometrics.tibs.org.

\section{ACKNOWLEDGEMENTS}

The authors thank G. Melville for providing the Dubbo weed data and A. Baddeley for questions and modification of the spatstat package. The authors also thank A. Zerbini and R. Hobbs for initial review of the article.

\section{REFERENCES}

Baddeley, A. and Turner, R. (2000). Practical maximum pseudolikelihood for spatial point patterns. Australian 85 New Zealand Journal of Statistics 42, 283-322.
Baddeley, A. and Turner, R. (2005). Spatstat: An R package for analyzing spatial point patterns. Journal of Statistical Software 12, $1-42$.

Berman, M. and Turner, T. R. (1992). Approximating point process likelihoods with GLIM. Applied Statistics 41, 31-38.

Borchers, D. L., Buckland, S. T., Goedhart, P. W., Clarke, E. D., and Hedley, S. L. (1998). Horvitz-Thompson estimators for doubleplatform line transect surveys. Biometrics 54, 1221-1237.

Borchers, D. L., Laake, J. L., Southwell, C., and Paxton, C. G. M. (2006). Accommodating unmodeled heterogeneity in doubleobserver distance sampling surveys. Biometrics 62, 372-378.

Buckland, S. T., Anderson, D. R., Burnham, K. P., and Laake, J. L. (1993). Distance Sampling: Estimating Abundance of Biological Populations. London: Chapman \& Hall.

Buckland, S. T., Breiwick, J. M., Cattanach, K. L., and Laake, J. L. (1993). Estimated population size of the California gray whale. Marine Mammal Science 9, 235-249.

Buckland, S. T., Anderson, D. R., Burnham, K. P., Laake, J. L., Borchers, D. L., and Thomas, L. (2001). Introduction to Distance Sampling: Estimating Abundance of Biological Populations. New York: Oxford University Press.

Buckland, S. T., Anderson, D. R., Burnham, K. P., Laake, J. L., Borchers, D. L., and Thomas, L. (2004). Advanced Distance Sampling: Estimating Abundance of Biological Populations. New York: Oxford University Press.

Buckland, S. T., Borchers, D. L., Johnston, A., Henrys, P. A., and Marques, T. A. (2007). Line transect methods for plant surveys. Biometrics 63, 989-998.

Burnham, K. P. and Anderson, D. R. (2002). Model Selection and Multimodel Inference: A Practical Information-theoretic Approach. New York: Springer-Verlag Inc.

Cox, D. R. (1955). Some statistical methods related with a series of events. Journal of the Royal Statistical Society, Series B 17, 129157.

Cressie, N. A. C. (1993). Statistics for Spatial Data. New York: John Wiley \& Sons.

Dawson, S. E., Slooten, S., DuFresne, S., Wade, P., and Clement, D. (2004). Small-boat surveys for coastal dolphins: line-transect surveys for Hector's dolphins (Cephalorhynchus hectori). Fishery Bulletin 102, 441-451.

Diggle, P. J. (2003). Statistical Analysis of Spatial Point Patterns, 2nd edition. London: Academic Press.

Dorfman, R. (1938). A note on the delta-method for finding variance formulae. The Biometric Bulletin 1, 129-137.

Eberhardt, L. L. (1967). Some developments in "distance sampling." Biometrics 23, 207-216.

Fewster, R. M., Buckland, S. T., Burnham, K. P., Borchers, D. L., Jupp, P. E., Laake, J. L., and Thomas, L. (2009). Estimating the encounter rate variance in distance sampling. Biometrics $\mathbf{6 5}$, 225-236.

Guan, Y. and Loh, J. M. (2007). A thinned block bootstrap variance estimation procedure for inhomogeneous spatial point patterns. Journal of the American Statistical Association 102, 1377-1386.

Hedley, S. L. and Buckland, S. T. (2004). Spatial models for line transect sampling. Journal of Agricultural, Biological, and Environmental Statistics 9, 181-199.

Innes, S., Heide-Jorgensen, M. P., Laake, J. L., Laidre, K. L., Cleator, H. J., Richard, P., and Stewart, R. E. A. (2002). Surveys of belugas and narwhals in the Canadian high Arctic in 1996. In Belugas in the North Atlantic and the Russian Arctic, M. P. HeideJorgensen and O. Wiig (eds), volume 4 of NAMMCO Scientific Publications, 169-190. Tromso, Norway: North Atlantic Marine Mammal Commission.

Laake, J. L. and Borchers, D. L. (2004). Methods for incomplete detection at distance zero. In Advanced Distance Sampling, 
S. Buckland, D. Anderson, K. Burnham, J. Laake, D. Borchers, and L. Thomas (eds), 108-189. New York: Oxford University Press.

Laake, J. L., Guenzel, R. J., Bengtson, J. L., Boveng, P. L., Cameron, M., and Hanson, M. B. (2008). Coping with variation in aerial survey protocol for line transect sampling. Wildlife Research $\mathbf{3 5}$, 289-298.

Marques, F. F. C. and Buckland, S. T. (2003). Incorporating covariates into standard line transect analyses. Biometrics 59, 924-935.

McCullagh, P. and Nelder, J. (1989). Generalized Linear Models, 2nd edition. Boca Raton, Florida: Chapman \& Hall/CRC.

Melville, G. J. and Welsh, A. H. (2001). Line transect sampling in small regions. Biometrics 57, 1130-1137.

Møller, J. and Waagepetersen, R. (2003). Statistical Inference and Simulation for Spatial Point Processes. Boca Raton, Florida: Chapman \& Hall/CRC.

Nayak, T. K. (2002). Rao-Cramer type inequalities for mean square error prediction. American Statistician 56, 102-106.

$\mathrm{R}$ Development Core Team. (2008). R: A language and environment for statistical computing. Vienna, Austria: R Foundation for Statistical Computing. ISBN 3-900051-07-0. http://www.Rproject.org, accessed April 23, 2009.

Ramsey, F. L. and Harrison, K. (2004). A closer look at detectability. Environmental and Ecological Statistics 11, 73-84.

Royle, J. A., Dawson, D. K., and Bates, S. (2004). Modeling abundance effects in distance sampling. Ecology 85, 1591-1597.

Schlather, M. (2001). Simulation of stationary and isotropic random fields. $R$ News $1,18-20$.
Schweder, T. (1977). Point process models for line transect experiments. In Recent Developments in Statistics, J. R. Barra, B. Van Cutsem, F. Brodeau, and G. Romier (eds), 221-242. Amsterdam: North Holland.

Skaug, H. (2006). Markov modulated Poisson processes for clustered line transect data. Environmental and Ecological Statistics 13, 199-211.

Thomas, L., Laake, J. L., Strindberg, S., Marques, F. F. C., Buckland, S. T., Borchers, D. L., Anderson, D. R., Burnham, K. P., Hedley, S. L., Pollard, J. H., Bishop, J. R. B., and Marques, T. A. (2006) Distance 5.0. U.K.: Research Unit for Wildlife Population Assessment, University of St. Andrews. http://www.ruwpa.st-and. ac.uk/distance/, accessed April 23, 2009.

Vidal, O., Barlow, J., Hurtado, L. A., Torre, J., Cendon, P., and Ojeda, Z. (1997). Distribution and abundance of the Amazon river dolphin ( $i$ nia geoffrensis) and the tucuxi (sotalia fluviatilis) in the Upper Amazon river. Marine Mammal Science 13, 427445.

Waagepetersen, R. and Schweder, T. (2006). Likelihood-based inference for clustered line transect data. Journal of Agricultural, Biological, and Environmental Statistics 11, 264279.

Wood, S. N. (2006). Generalized Additive Models: An introduction with $R$. Boca Raton, Florida: Chapman and Hall/CRC.

Received July 2008. Revised January 2009. Accepted January 2009. 